\title{
Téoros
}

Revue de recherche en tourisme

\section{Les défis de la forêt privée}

La conservation, l'utilisation durable de la forêt et

l'écotourisme

\section{Louise Gratton et Francine Hone}

Volume 25, numéro 3, automne 2006

Tourisme et forêt, visions d'une gestion intégrée

URI : https://id.erudit.org/iderudit/1071016ar

DOI : https://doi.org/10.7202/1071016ar

Aller au sommaire du numéro

Éditeur(s)

Université du Québec à Montréal

ISSN

0712-8657 (imprimé)

1923-2705 (numérique)

Découvrir la revue

Citer cet article

Gratton, L. \& Hone, F. (2006). Les défis de la forêt privée : la conservation, l'utilisation durable de la forêt et l'écotourisme. Téoros, 25(3), 30-35.

https://doi.org/10.7202/1071016ar d'utilisation que vous pouvez consulter en ligne.

https://apropos.erudit.org/fr/usagers/politique-dutilisation/ 


\section{Les défis de la forêt privée La conservation, I'utilisation durable de la forêt et l'écotourisme}

\section{Louise Gratton et Francine Hone}

Aux cours des dernières années, le sort de la forêt publique, principalement boréale, a été au cœur du débat sur la gestion de la forêt québécoise. Pourtant les enjeux de la conservation de la biodiversité sont bien plus grands dans la forêt méridionale qui, dans une large part, est de tenure privée. C'est ici que se concentre la richesse spécifique du territoire québécois, où le plus grand nombre d'animaux et de plantes sont en situation précaire, où l'érosion des sols et la détérioration de la qualité de l'eau engendrent les plus grands conflits, où les avantages socioéconomiques que procure la forêt aux populations locales sont les plus menacés (Corridor appalachien, 2003). Tout comme pour les forêts publiques, le maintien de la productivité des écosystèmes forestiers, la conservation de la diversité biologique et le respect des besoins des populations locales constituent, en forêt privée, les assises incontournables sur lesquelles la gestion du territoire forestier devrait dorénavant s'appuyer.

La forêt privée occupe au Québec 70400 kilomètres carrés, soit $11 \%$ du territoire forestier productif, et appartient à quelque 130000 propriétaires de lots boisés. La presque totalité (95\%) de cette superficie forestière est occupée par des forêts feuillues et mélangées, tandis que seuls $5 \%$ sont occupés par des peuplements résineux. La forêt privée se trouve principalement dans les régions des Laurentides, de l'Estrie, de l'Outaouais, de la ChaudièreAppalaches, de la Mauricie-Bois-Francs, de la Montérégie et du Bas-Saint-Laurent. L'apport des pratiques forestières en terre privée représente $20 \%$ de l'approvisionnement en bois rond des usines de transfor- mation et les activités en forêt privée génèrent chaque année des revenus annuels de quelque $800 \mathrm{M} \$$, dont $500 \mathrm{M} \$$ proviennent de la récolte de bois (MRNF, 2003).

Les apports économiques de la forêt privée ne se limitent pas aux seules pratiques forestières visant à approvisionner l'industrie ; la production de bois de chauffage et de produits de l'érable, tout comme la culture d'arbres de Noël, constituent d'autres activités importantes qui génèrent des revenus aux propriétaires de lots boisés. La chasse et la pêche le sont tout autant, puisque, dans $50 \%$ des cas, c'est sur les terres privées que s'exercent ces deux activités (MRNF, 2003). Bien que les retombées se manifestent davantage auprès des communautés que des propriétaires individuels, à elles seules ces deux activités dans les régions où dominent les terres privées génèrent, selon le ministère des Ressources naturelles et de la Faune (MRNF, 2003), des revenus de $510 \mathrm{M} \$$ pour la pêche sportive et de $131 \mathrm{M} \$$ pour la chasse. Toujours selon cette même source, la contribution des activités sans prélèvement et de plein air est évaluée dans son ensemble à $708 \mathrm{M} \$$.

L'exploitation forestière, les ressources non ligneuses de la forêt, les activités cynégétiques, halieutiques et de plein air, la présence de la flore et de la faune et la beauté du paysage sont donc intimement liées au développement économique des communautés locales. Plusieurs régions ont depuis longtemps reconnu le fait que la diversification des revenus de la forêt est économiquement souhaitable et qu'un aménagement et une utilisation polyvalents et durables des ressources forestières sont critiques à la survie des communautés locales et contribuent largement à la qualité de vie des résidants de la région.
Bien qu'indissociables de certaines ressources ou valeurs dont «l'exploitation » génère des retombées économiques directes, la conservation de la diversité biologique est globalement moins tangible et parfois plus difficilement quantifiable.

Un grand nombre d'espèces de la flore et de la faune, dont plusieurs à statut précaire, dépendent entièrement ou en partie des milieux forestiers. À titre d'exemple, on considère que plus de $90 \%$ des espèces aviaires nord-américaines menacées dépendent des habitats forestiers (American Forest Foundation, 2006) et que $12 \%$ de la flore menacée ou vulnérable au Québec y trouve refuge.

Au-delà des seules préoccupations floristiques et fauniques, les forêts procurent des bénéfices indirects. Elles jouent un rôle-clé dans le maintien de la qualité du milieu hydrique dont l'altération peut occasionner, entre autres, des menaces pour la santé et la sécurité publique, une augmentation des coûts liés au traitement de l'eau potable, une détérioration de la valeur patrimoniale et des activités récréotouristiques. Le maintien d'écosystèmes forestiers en santé signifie également la préservation de la structure et de la texture du sol, d'un couvert adéquat, du taux de matière organique ou d'autres propriétés importantes à la pérennité de la ressource forestière, comme la rétention de l'eau et la survie des organismes du sol. Finalement, la reconnaissance du rôle des forêts dans la séquestration du carbone devient un enjeu bien réel dans le débat sur les changements climatiques.

André Bouchard (dans Cantin et Potvin, 1996) reconnaît cependant que peu de chercheurs se sont penchés sur les relations entre d'une part les processus écologiques, qui relèvent des sciences naturelles, et d'autre part les im- 
pacts socioécomiques de différentes stratégies d'utilisation de la forêt, qui relèvent des sciences sociales. Selon cet auteur, la difficile mais stimulante responsabilité de conserver et de mettre en valeur le patrimoine forestier, l'une des plus grandes ressources naturelles et économiques, doit être partagée par l'ensemble des citoyens, autant les citadins que ceux qui vivent en région.

Le défi est de taille et d'autant plus grand en forêt privée puisque, entre les utilisateurs de plus en plus nombreux, la reconnaissance des enjeux écologiques, économiques et sociaux n'est pas nécessairement partagée, voire comprise. Un consensus se dégage cependant : malgré le rôle central que joue la présence des milieux forestiers dans l'économie locale et le bien-être des individus, tous les intervenants reconnaissent que la forêt subit des menaces importantes provenant de la conversion des terres forestières en faveur de l'étalement urbain, du développement domiciliaire et de villégiature, du développement d'infrastructures routières, de certains usages récréatifs intensifs, de même que des pratiques forestières qui ne tiennent pas compte des impacts négatifs sur la biodiversité et la pérennité de la ressource.

En plus de perdre des milieux forestiers et de diminuer la valeur écologique (fragmentation des milieux naturels, perte de diversité biologique, invasions d'espèces exotiques, etc.) qui leur est associée, il s'ensuit aussi une perte "des services et des produits » à caractère économique, associée directement à l'exploitation de la matière ligneuse, mais également à l'utilisation de la forêt à d'autres fins.

Une utilisation inappropriée du milieu et le développement d'activités non respectueuses de l'environnement peuvent avoir sur une communauté des impacts négatifs, tant du point de vue écologique que social et économique (Silva Forest Foundation, 1997).

Dans une optique de préserver les écosystèmes forestiers, les processus écologiques et les espèces qui en dépendent, tout en prenant en compte les besoins des populations locales, il importe de travailler à différentes échelles de perception, c'est-à-dire de privilégier une planification de la conservation à l'échelle du paysage, de prendre en compte la protection des ressources forestières à l'échelle de l'écosystème et d'impliquer les propriétaires à l'échelle de leurs lots boisés.

\section{La planification à l'échelle du paysage}

La planification stratégique à l'échelle du paysage s'appuie sur les principes de la biologie de la conservation. Cette discipline a vu le jour à la fin des années 1970 lorsque des scientifiques, notamment l'écologiste Michael Soulé, prirent conscience que la crise éminente à laquelle faisait face la diversité biologique ne pourrait être résolue qu'en combinant les expertises pratiques de la gestion de la faune et des forêts avec les théories de la biologie des populations et de la biogéographie. Le résultat fut le développement de nouvelles approches et de méthodes pour, notamment, préserver les espèces (Primack, 1998).

Devant le constat d'échec des aires protégées dans la préservation de populations viables de grands carnivores et herbivores (Meffe et Carroll, 1994), les premières applications pratiques en biologie de la conservation ont porté sur l'établissement, le design et la gestion des aires naturelles. Ainsi, la planification de la conservation à l'échelle du paysage s'appuie sur :

- l'identification de noyaux de conservation, c'est-à-dire des aires de superficie assez vaste pour assurer la protection et la survie des populations d'espèces re- présentatives de la région naturelle et protéger tous les écosystèmes et les processus écologiques qui les maintiennent :

- l'établissement de zones "tampons" autour de ces noyaux qui, tout en permettant des usages multiples, ne mettent pas en péril leur intégrité écologique ou comportent des mesures pour les rendre compatibles avec la protection des espèces et des habitats les plus sensibles ;

- l'identification de corridors faisant partie intégrante de la planification à l'échelle du paysage, de manière à assurer la connectivité entre les milieux naturels, favorisant ainsi la migration des animaux d'un fragment forestier à un autre et, par le fait même, les échanges génétiques et la recolonisation après une extinction locale. Ces corridors facilitent aussi les déplacements en accroissant l'étendue des aires d'alimentation et offrent des abris contre les prédateurs; ils tiendraient également lieu de refuge advenant des perturbations ou la disparition des habitats davantage privilégiés. Finalement, ils procurent aux espèces qui le requièrent l'accès à des territoires plus grands ainsi qu'à une diversité de milieux naturels (fig. 1).

\section{Figure 1}

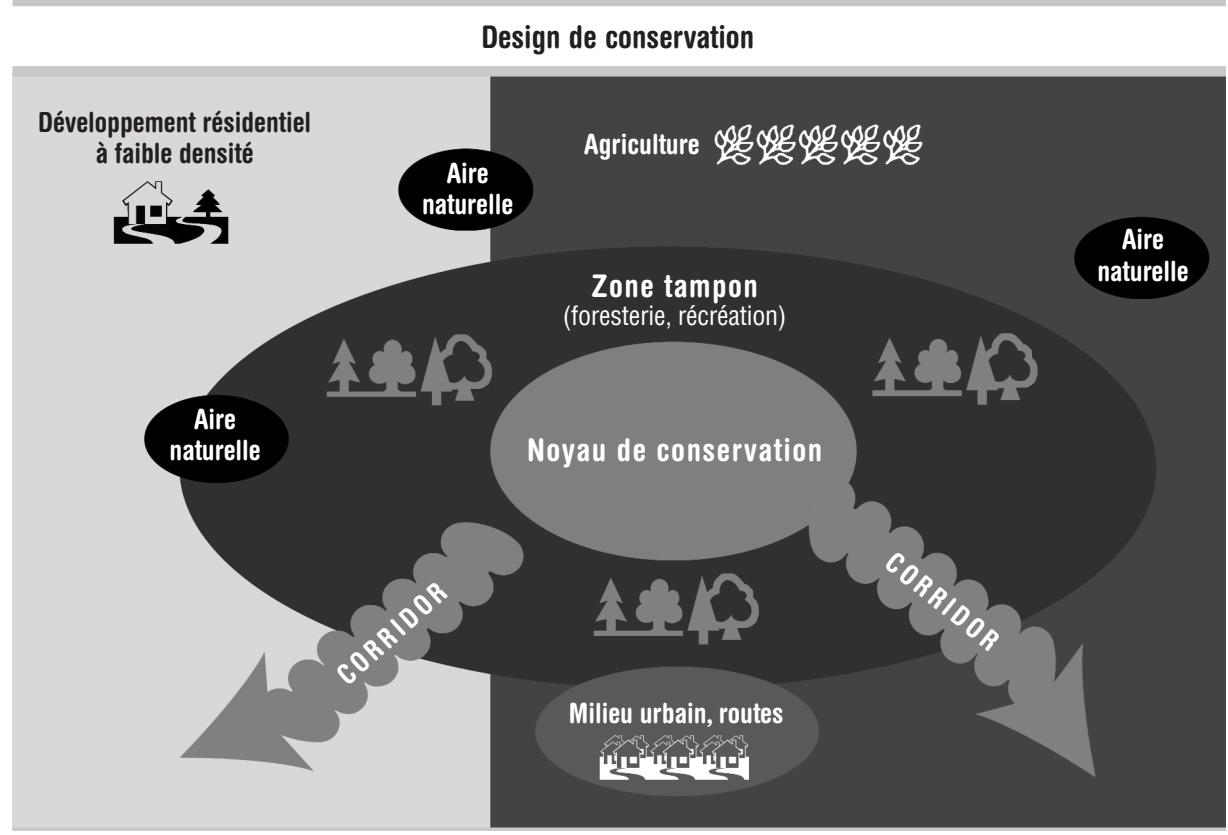

Source: The Wildlands Project. 


\section{La protection des écosystèmes forestiers}

Utilisant comme cadre de référence la planification à l'échelle du paysage, il importe de prendre en compte, à l'échelle des écosystèmes, la connaissance de ces milieux, ce qui permettra de guider leur protection et de maintenir leurs processus écologiques. Pour ce qui est de l'écosystème forestier, ce type de planification est un outil qui permet d'assurer la viabilité de la forêt ainsi que les multiples usages qui en découlent. Cette planification vise en premier lieu l'acquisition de la connaissance écologique du territoire et définit par la suite les usages anthropiques qui peuvent être développés et maintenus de manière durable à l'intérieur des limites écologiques de l'écosystème. Le maintien des écosystèmes forestiers et de la diversité biologique permet ainsi d'assurer les activités économiques générées par ces mêmes écosystèmes.

La planification des activités humaines qui intègrent la protection, le maintien et, dans certains cas, la restauration des écosystèmes est considérée par plusieurs comme étant à la base du développement économique durable. Un des principes qui sous-tend ce type de planification à l'échelle de l'écosystème dicte l'importance d'une telle approche pour l'économie locale. Pour développer une économie durable, on doit favoriser une diversité d'activités qui répondent aux besoins des individus et de la communauté, tout en assurant la protection et le maintien du capital naturel. Des communautés en santé dépendent et soutiennent des écosystèmes diversifiés et en santé (Silva Forest Foundation, 1997).

\section{La participation des propriétaires}

La tenure privée de la majorité du territoire forestier du Québec méridional fait en sorte que la conservation et la gestion du milieu forestier doivent être abordées de manière distincte de celle appliquée à la forêt publique et favoriser l'engagement du propriétaire dans une démarche basée sur le respect de son droit de propriété et de ses besoins, tout en tenant compte d'une approche plus globale à l'échelle de l'écosystème et du paysage.

La participation volontaire des propriétaires fonciers, qu'il s'agisse de producteurs forestiers, de compagnies forestières ou de simples propriétaires de terres boisées, est essentielle pour envisager la protection à perpétuité des milieux naturels sur le territoire. Depuis quelques années, on remarque un nombre grandissant de propriétaires de milieux forestiers qui sont conscientisés à l'importance de préserver leur forêt, mais qui veulent aussi maintenir certains usages. En Estrie, cette nouvelle tendance n'est pas inconnue au fait qu'il existe un nombre croissant d'organismes de conservation locaux ou régionaux dont la vocation première est la protection à perpétuité des milieux naturels. Ces organismes offrent un important soutien aux propriétaires qui désirent s'engager dans la préservation de leur forêt par le biais d'ententes de conservation, comme le don écologique de leur propriété ou d'une partie de leur propriété à un organisme de conservation, la servitude de conservation, ou encore en désignant leur propriété à titre de réserve naturelle. En contrepartie à l'engagement des propriétaires dans une telle démarche menant à une entente légale, ceux-ci ont accès à des mesures incitatives fiscales ou à des rabais de taxes foncières.

\section{Nouveaux défis}

Les écologistes reconnaissent aujourd'hui que l'objectif de $12 \%$ de territoires protégés fixé, bien aléatoirement, par la communauté internationale (Brundtland, 1988) ne suffira pas à protéger la diversité biologique et à préserver les espèces de l'extinction (Groves, 2003). Noss et Cooperrider (1994) estiment de $25 \%$ à $75 \%$ la proportion d'une région qui devrait être protégée pour atteindre les objectifs de conservation de la biodiversité. L'écart s'explique par l'étendue et la diversité des types d'habitats représentés dans une région donnée et n'exprime pas nécessairement l'exclusion d'usages qui dépend davantage de la vulnérabilité des habitats et des espèces ciblées pour la conservation.

Sur un total de près de 130000 propriétaires de lots boisés, ce sont, chaque année, 30000 propriétaires enregistrés qui vendent du bois. Selon plusieurs intervenants forestiers, il s'agit d'un potentiel inexploité et une hausse de la possibilité forestière pourrait contribuer davantage à l'activité économique régionale et amenuiser les impacts des aléas du marché et de la valeur du dollar canadien qui rendent incertaine dans certaines régions la survie de plusieurs entreprises de transformation du bois.
On reconnaît qu'une partie importante de l'industrie touristique dépend d'un environnement naturel de qualité (Ministère du Tourisme du Québec, 2005). Une analyse de D.K. Foot (2000) renforce la portée de cette affirmation à l'effet que, partout dans le monde, la tendance vers les activités liées à la nature et au plein air va en s'amplifiant. D'ailleurs, la projection du taux de croissance de 1996 à 2011 pour l'observation des oiseaux est de $6 \%$, devant le golf (3\%) et la pêche sportive (4,5\%).

Ce sont là des constats bien réels et les perceptions fragmentées, voir biaisées entre les divers utilisateurs du milieu forestier, le sont tout autant. Sources potentielles de conflits, elles ont pourtant en commun la préservation du patrimoine forestier. De nouvelles pistes sont à explorer.

\section{Nouvelles pistes à explorer}

Jusqu'à maintenant, la conservation des ces milieux naturels en terre privée s'est effectuée sans qu'il y ait nécessairement de conflits d'usages comme la coupe forestière ou les activités touristiques. Cependant, comme l'utilisation de la forêt à des fins d'exploitation commerciale est d'une importance économique capitale pour les petites communautés locales et qu'il existe un grand nombre de propriétaires forestiers et d'intervenants du secteur forestier qui dépendent presque exclusivement de l'exploitation forestière, d'autres avenues doivent être explorées afin d'atteindre à la fois les objectifs de conservation et ceux d'utilisation de la forêt.

\section{Conservation volontaire}

Pour que la conservation sur les terres privées prenne l'ampleur requise pour maintenir la biodiversité et pour atteindre les objectifs de conservation, que ce soit ceux de la Stratégie québécoise sur les aires protégées (8\%) ou de la Commission des Nations unies sur l'environnement et le développement (12\%), la contribution des groupes de conservation à l'échelle nationale, régionale ou locale est indispensable. Au Québec, cependant, on compte moins de 20 organismes dont la vocation première est la conservation volontaire. En NouvelleAngleterre seulement, ils sont plus de 200. Leur rôle va de la promotion de la conservation auprès des propriétaires de lots boisés à l'acquisition de terres de haute valeur 
écologique, soit par l'achat, l'acceptation de dons ou de servitudes de conservation. L'organisme Conservation de la nature Canada (CNC) est le chef de file dans ce domaine et, aux cours des années, il a fait l'acquisition de plusieurs sites de haute valeur écologique représentant au Québec quelque 14000 hectares.

Par ailleurs, l'impact sur les plans local et régional d'une planification de la conservation à l'échelle du paysage peut être très significatif. L'exemple de Corridor appalachien (voir encadré 1) est éloquent à cet égard. La stratégie de conservation proposée par cet organisme a considérablement accéléré la création d'aires naturelles protégées. En 2000, il n'existait qu'une aire protégée de superficie appréciable dans la région: le parc national du Mont-Orford (58,4 km). Dans le passé, plusieurs organismes de conservation régionaux et des fiducies foncières locales avaient réussi à protéger environ 400 hectares (1000 acres) de milieux naturels. La mise en œuvre de son projet de conservation du Corridor appalachien, en collaboration avec ses membres affiliés et ses partenaires, a vu la superficie des aires protégées augmenter à plus de 6400 hectares (16 000 acres). Près des deux tiers du massif des monts Sutton, soit environ 60 kilomètres carrés, sont maintenant protégés à perpétuité, ce qui représente la plus grande aire de conservation privée à l'est de la Saskatchewan (fig. 2).

\section{Servitudes de conservation forestière}

En principe, les critères d'un aménagement forestier durable, tel que le dicte le préambule de la Loi sur les forêts, devraient permettre de répondre à ces nouveaux défis, c'est-à-dire : 1) la conservation de la biodiversité, 2) le maintien de la productivité et de la santé des écosystèmes, 3) la conservation des sols et des eaux, 4) le maintien de l'apport des écosystèmes forestiers aux grands cycles écologiques, 5) le maintien des avantages socioéconomiques multiples que procurent les forêts à la société et 6) la prise en compte, dans les choix de développement, des valeurs et des besoins des populations concernées.

Or, selon plusieurs intervenants à la Commission d'étude sur la gestion de la forêt publique québécoise (2004), les indicateurs d'atteinte de ces critères qui respectent un

Figure 2

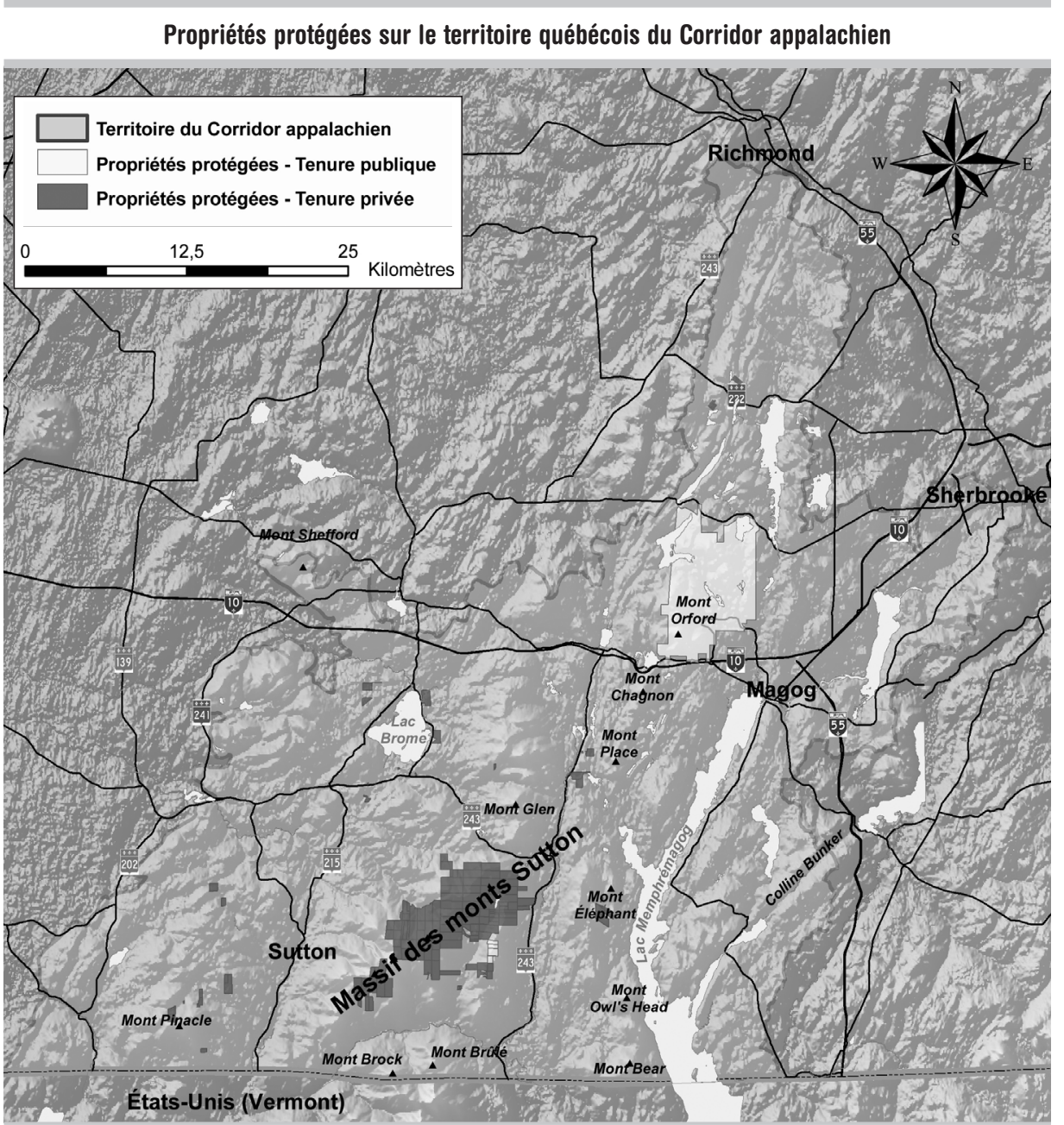

Source: Corridor appalachien (ACA) à partir de l'information géographique de référence provenant de la base de données topographiques du Ministère des ressources naturelles et faune (MRNF), novembre 2005.

\section{Encadré 1}

\section{L'exemple du Corridor appalachien}

Le Corridor appalachien est un de ces organismes de conservation d'envergure régionale qui a pour mission de protéger les milieux naturels de la région des Appalaches. Par le biais d'une stratégie de conservation transfrontalière qu'il a mise de l'avant, cet organisme procure aux collectivités locales des Cantons-de-l'Est les moyens de maintenir et de restaurer un cadre de vie qui respecte l'écologie de la région dans une perspective de développement durable. Le Corridor appalachien offre une assistance technique, des conseils et de l'expertise aux organismes de conservation et aux intervenants qui partagent sa vision.

Le territoire sur lequel œuvre le Corridor appalachien comprend un segment de la chaîne des Appalaches qui chevauche la frontière canadoaméricaine. Au Québec, ce territoire couvre 241900 hectares (approximativement 597700 acres) et renferme le massif des monts Sutton et ses contreforts, le bassin versant du lac Memphrémagog, ainsi que des sites périphériques tels le mont Pinacle et le lac Brome. II rejoint le mont Orford et la rivière Saint-François. $\mathrm{Au}$ Vermont, il correspond à la section des Montagnes vertes qui s'étend au sud jusqu'au mont Mansfield et au Camel's Hump.

La stratégie de conservation mise de l'avant par le Corridor appalachien se base sur la protection de noyaux de conservation comme celui du massif des monts Sutton, ainsi que l'établissement de zones «tampons » autour de ces noyaux et des corridors fauniques qui assurent la connectivité entre ces noyaux de conservation.

Pour en savoir davantage, consulter: [wWw. apcor.ca]. 
aménagement forestier durable sont loin de faire la démonstration du développement durable. Ce même constat peut être fait pour la forêt privée où, en raison du nombre d'intervenants forestiers (propriétaires individuels, regroupements, agences et municipalités), l'application de ces critères est encore plus complexe.

Une planification de la conservation basée sur une approche écosystémique pourrait permettre l'application concrète des principes du développement durable. En identifiant les éléments sensibles du territoire qui méritent d'être soustraits au développement forestier et en guidant le propriétaire forestier vers une utilisation durable de la forêt, il serait possible à la fois d'assurer la préservation de milieux d'intérêt écologique et l'utilisation durable de la forêt essentielle pour l'économie régionale. Cette démarche doit cependant être intimement liée au soutien de ceux qui travaillent dans la forêt et l'utilisent ainsi que des divers intervenants du secteur forestier. Pour amener ces nouveaux joueurs à participer concrètement à la conservation de la biodiversité, il faut être en mesure de proposer des moyens ou des outils qui respecteront à la fois les objectifs de conservation et ceux de la pérennité de la ressource forestière.

Pour concrétiser cette approche, certains organismes de conservation américains proposent aux propriétaires de terres forestières une servitude de conservation forestière (working forest easement). Cet outil de conservation mériterait qu'on s'y attarde davantage, car il est basé sur le maintien de la forêt comme ressource exploitable, mais également sur le maintien de l'écosystème naturel et des processus écologiques ainsi que des activités secondaires qui en découlent, comme la chasse, la pêche, le paysage et l'écotourisme. La servitude, qui est une entente légale, permet donc d'assurer la pérennité de la ressource forestière et des milieux naturels en tenant compte des objectifs du propriétaire et de l'organisme de conservation.

Cet outil, qui serait une avenue intéressante pour le Québec, permettrait d'assurer que la vocation forestière soit maintenue à perpétuité sans qu'on puisse la convertir à d'autres fins ou subdiviser la propriété pour le développement domiciliaire ou de villégiature, une situation qui menace la pérennité des superficies forestières exploitables dans plusieurs régions du Québec. Depuis 1990 dans les États de la NouvelleAngleterre (Vermont, New Hampshire et Maine), 500000 acres ont été acquis à des fins de conservation et 2 millions d'acres ont été désignés working forest easement dans les zones tampons autour de ces aires de conservation (Ginn, 2005). À cela s'ajoutent 8 millions d'acres de forêts certifiées, une autre avenue sous-utilisée en forêt privée.

\section{Certification forestière}

La certification écoforestière est un processus qui consiste à évaluer la bonne gestion forestière, c'est-à-dire une gestion qui tient compte de l'environnement et des impacts sociaux tout en étant économiquement viable. La certification apporte des avantages indéniables aux différents propriétaires adhérents : de meilleures relations avec la collectivité, une contribution à la pérennité des forêts, le maintien des marchés, une amélioration de l'image corporative grâce à la reconnaissance internationale (Legris, 2003).

Les produits qui proviennent d'une forêt certifiée peuvent être identifiés par une étiquette démontrant que la forêt est bien gérée. Une telle identification permet d'assurer aux consommateurs, aux détaillants, aux gouvernements et aux investisseurs l'origine des produits (Forest Stewardship Council, 2004). Un certificat de chaîne de " traçabilité » permet aussi le suivi du bois de source certifié depuis son origine jusqu'au consommateur.

À ce jour, au Canada, les normes de certification ont été développées pour la forêt boréale et les normes relatives aux forêts feuillues et mélangées sont sur le point d'être adoptées. Par contre, les systèmes de certification actuels concernent davantage les grandes forêts et peu sont adaptés aux petites forêts de moins de 4000 hectares (Carbonneau, 2005). À moins d'être de grands propriétaires terriens ou de se réunir en coopérative, les propriétaires de petits lots boisés ont peu accès à la certification. Pour pallier cette situation, un programme pancanadien de certification des forêts privées est en développement.

\section{Diversification des produits et services}

Par ailleurs, certains auteurs croient qu'un développement économique durable et régional de la forêt qui permet de maintenir la ressource forestière tout en préservant l'écosystème doit tenir compte de la diversification des produits. Selon eux, il faut percevoir la forêt non pas uniquement comme une source de bois de sciage et de pâte, mais davantage comme une source économique variée basée sur la production d'une multitude de produits et services (Phillips, 1994). II peut s'agir de produits acéricoles, de plantes comestibles ou médicinale, de champignons et de services comme les activités récréatives et touristiques (Table ronde sur l'environnement et l'économie, 2005).

\section{L'écotourisme comme outil de conservation}

De plus en plus de communautés locales développent un créneau touristique basé sur la beauté des paysages et dépendent de cette ressource pour le développement de cette industrie.

Partout en Amérique du Nord il y a, depuis quelques années, un intérêt marqué pour le tourisme de nature et l'écotourisme. Ce type de tourisme offre des avantages potentiels considérables aux communautés qui leur sont associées. Des régions situées à proximité des grand centres urbains, comme celle des Appalaches, reçoivent un nombre croissant d'écotouristes attirés par la beauté des paysages et la présence de milieux forestiers encore «sauvages". Mis à part les parcs de conservation et les autres aires naturelles protégées sur des terres publiques, il existe très peu d'endroits accessibles à ce type d'utilisateur.

Dans les Cantons-de-l'Est, certaines initiatives mises de l'avant depuis plusieurs années par des organismes sans but lucratif facilitent l'accès à un important réseau de sentiers pédestres. Ces sentiers aménagés en forêt se trouvent en grande partie sur des terres privées et nécessitent de la part de ces organismes des ententes de droits de passage avec les propriétaires. Ces infrastructures assurent depuis de nombreuses années des "services récréatifs ». Toutefois, pour assurer "l'industrie durable » qu'est l'écotourisme, il faut nécessairement s'assurer du maintien de la ressource dont elle dépend, notamment des milieux forestiers, de l'accessibilité et de la qualité du paysage. 
Dans ce contexte, le tourisme de loisirs basé sur la nature est un important moyen de trouver, auprès du grand public, un appui à la conservation du patrimoine naturel et d'améliorer sa sensibilisation aux valeurs multiples et importantes de la forêt sur les plans écologique, culturel, spirituel, esthétique, récréatif et économique, en plus de générer un revenu pour les activités de conservation. Le tourisme doit aussi contribuer à améliorer la qualité de vie des communautés locales et inciter le public à soutenir les valeurs traditionnelles, à protéger et à respecter les milieux naturels (Wight, 1997).

Les coûts écologique, social et culturel du tourisme peuvent néanmoins être considérables. Même des impacts limités peuvent avoir une incidence majeure sur la conservation du patrimoine naturel. Le tourisme, même l'écotourisme, s'il n'est pas dûment planifié, conçu et géré, peut contribuer à la détérioration des paysages et menacer la biodiversité et la dégradation des écosystèmes.

\section{Encadré 2}

\section{Vision d'avenir de}

\section{la forêt québécoise}

"Les forêts québécoises sont une richesse commune et appréciée pour chacune des valeurs qu'elles représentent. Elles sont un ensemble diversifié d'écosystèmes qui produisent d'importantes ressources renouvelables, sources de qualité de vie pour les gens, particulièrement les Premières Nations pour lesquelles les forêts sont le terroir de leurs cultures. Elles jouent un rôle primordial au point de vue social, environnemental et économique, tant pour les régions que pour l'ensemble de la société québécoise. Elles fournissent la matière première à une industrie vigoureuse qui se positionne favorablement sur le marché mondial. Elles sont un vaste milieu naturel à la base d'activités récréotouristiques, de villégiature et de plein air. À l'échelle des paysages, elles forment une mosaïque complexe et en constante évolution. Elles sont au cœur des cycles de l'eau et du carbone. Elles sont riches de vie faunique et floristique. De la diversité et de la qualité de ces écosystèmes forestiers dépendent directement leur capacité de fournir l'ensemble des bienfaits auxquels les Québécoises et Québécois sont en droit de s'attendre. »

Commission d'étude sur la gestion de la forêt publique québécoise, 2004

\section{Conclusion}

Concilier la conservation de la biodiversité, l'utilisation durable de la forêt et le tourisme est réalisable en autant que l'on mette en place une planification qui tienne compte des besoins des écosystèmes forestiers et des besoins économiques et sociaux des régions. Cette assertion se reflète dans la vision de l'avenir de la forêt québécoise dégagée par la Commission Coulombe (voir encadré 2).

Selon W.J. Ginn (2005), le message est simple: il faut planifier la conservation du patrimoine naturel à l'échelle du paysage tout en reconnaissant que les communautés sont organisées autour de systèmes économiques. L'utilisation durable de la ressource forestière sous toutes ses formes, si elle est bien planifiée, structurée et soutenue adéquatement par des incitatifs, est probablement la seule alternative possible pour maintenir les communautés en harmonie avec la biodiversité de la planète.

Louise Gratton est écologiste, directrice des sciences et de la gestion des milieux naturels, Conservation de la nature Canada, région du Québec.

Francine Hone est biologiste en conservation et directrice générale du Corridor appalachien.

\section{Bibliographie}

American Forest Foundation (2006), Forested Flyways, [http://www.affoundation.org].

Brundtland, G.H. (1988), Notre avenir à tous. La commission mondiale sur l'environnement et le développement, Montréal (Québec), Éditions du fleuve.

Cantin, D., et C. Potvin (1996), L'utilisation durable des forêts québécoises, de l'exploitation à la protection, Ste-Foy, Les Presses de I'Université Laval.

Commission d'étude sur la gestion de la forêt publique québécoise (2004), Résumé du rapport final, [http://www.commission-foret.qc. $\mathrm{ca} /$ rapportfinal.htm].

Carbonneau, S.-E. (2005), La certification forestière au sein des petites forêts privées, Syndicat des producteurs de bois de l'Estrie.

Corridor appalachien (2003), Mémoire présenté par le Corridor appalachien à la Commission d'étude sur la gestion de la forêt publique québécoise, [http://www.commission-foret. qc.ca/avis-region.htm\#ecqm].
Foot, D.K. (2000), Boom, Bust and Echo. Profiting from the Demographic Shift in the $21^{\text {st }}$ Century, Toronto, Stoddart Publishing.

Forest Stewardship Council (2004), FSC Principles and Criteria, [http.www.fsccanada.org].

Ginn W.J. (2005), Investing in Nature, Case Studies of Land Conservation in Collaboration with Business, Washington, DC, Island Press.

Groves, C.R. (2003), Drafting a Conservation Blueprint. A Practitioner's Guide to Planning for Biodiversity, Washington, DC, Island Press.

Legris, J. (2003), «En avant avec la certification forestière ", Info forêt, no 79, Ministère des Ressources naturelles, de la Faune et des Parcs.

Lind, B. (2001), Working Forest Conservation Easements, Land Trust Alliance, Washington, DC.

Meffe, G.K, et C.R. Carroll (1994), Principles of Conservation Biology, Sunderland, MA, Sinauer Associates.

Ministère des Ressources naturelles et de la Faune (MRNF), 2003, [http://www.mrnf.gouv. qc.ca/forets/privees/index.jsp].

Ministère du Tourisme du Québec (2005), Vers un tourisme durable. Politique touristique du Québec.

Noss, R.F., et A.Y. Cooperrider (1994), Saving Nature's Legacy: Protecting and Restoring Biodiversity, Washington, DC, Island Press.

Phillips, S.R. (1994), The Northern Forest Strategies for Sustainability, Volume 2: Development Opportunities for the Maine Woods, Washington, DC, The Wilderness Society.

Primack, R.B. (1998, $2^{\text {nd }}$ ed.), Essentials of Conservation Biology, Sunderland, MA, Sinauer Associates.

Silva Forest Foundation (1997), An Ecosystembased Approach to Forest Use: Definition and Scientific Rationale, [http://www.silvafor. org].

Table ronde nationale sur l'environnement et l'économie (TRNEE), 2005. Avenir boréal: La gouvernance, la conservation et le développement dans la région boréale du Canada, [http:// www.nrtee-trnee.ca].

Wight, P. (1997), Sustainability, Profitability and Ecotourism Markets: What Are They and How Do They Relate?, Pamela Wight \& Associates, Canada International Conference on Central and Eastern Europe and Baltic Sea Region, Pärnu, Estonie. 